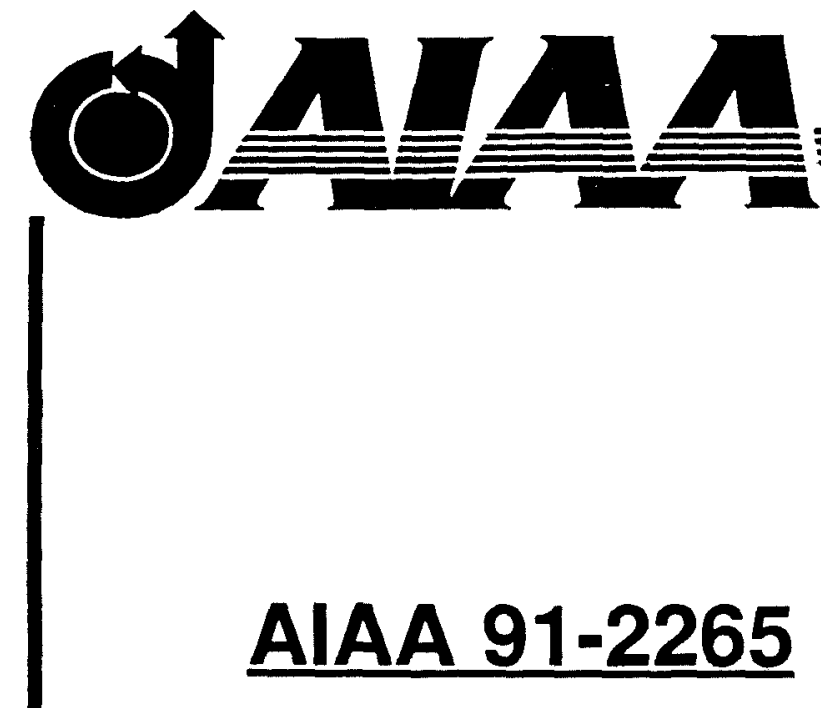

\title{
An Investigation of a Contoured Wall Injector for Hypervelocity Mixing Augmentation
}

I. A. Waitz, F. E. Marble, and E. E. Zukoski

California Institute of Technology

Pasadena, California 91125

\section{AIAA/SAE/ASME 27th Joint Propulsion Conference June 24-26, 1991 / Sacramento, CA}




\title{
AN INVESTIGATION OF A CONTOURED WALL INJECTOR FOR HYPER VELOCITY MIXING AUGMENTATION
}

\author{
Ian A. Waitz, ${ }^{*}$ Frank E. Marble, ${ }^{* *}$ and Edward E. Zukoski ${ }^{* * *}$ \\ California Institute of Technology, Pasadena, California 91125
}

\begin{abstract}
An experimental and computational investigation of a contoured wall fuel injector is presented. The injector was aimed at enabling shock-enhanced mixing for the supersonic combustion ramjet engines currently envisioned for applications on hypersonic vehicles. Three-dimensional flow field surveys, and temporallyresolved planar Rayleigh scattering measurements are presented for Mach 1.7 helium injection into Mach 6 air. These experimental data are compared directly with a three-dimensional Navier-Stokes simulation of the flow about the injector array. Two dominant axial vorticity sources are identified and characterized. The axial vorticity produced strong convective mixing of the injectant with the freestream. Shock-impingement was particularly effective as it assured seeding of baroclinic vorticity directly on the helium/air interface. The vorticity coalesced into a counter-rotating vortex pair of a sense which produced migration of the helium away from the wall. The influences of spatial averaging on the representation of the flow field as well as the importance of the fluctuating component of the flow in producing molecularly-mixed fluid are addressed.
\end{abstract}

* Graduate Student in Aeronautics, now Assistant Professor of Aeronautics and Astronautics, Massachusetts Institute of Technology

** Professor of Mechanical Engineering and Jet Propulsion, Emeritus, Fellow AIAA

*** Professor of Mechanical Engineering and Jet Propulsion, Fellow AIAA

Copyright (c) 1991 American Institute of Aeronautics and Astronautics, Inc. All rights reserved.
Nomenclature

$\overline{\mathrm{A}}$ injectant to freestream area ratio

$\mathrm{c}_{\mathrm{He}}$ helium mass fraction

D binary diffusion coefficient

E hot wire anemometer output

$h_{i}$ height of injection plane

$\bar{m}$ injectant to freestream molecular weight ratio

$\overline{\dot{m}}$ injectant to freestream mass flux ratio

M Mach number

$\mathrm{M}_{\infty}$ freestream Mach number

p static pressure

$\mathrm{p}_{\mathrm{c}}$ cone static pressure

$\mathrm{p}_{\mathrm{t}}$ pitot pressure

$p_{0}$ total pressure

$\mathrm{p}_{\infty}$ freestream static pressure

$\overline{\mathrm{p}}$ injectant to freestream static pressure ratio

Re Reynolds number

$t$ time

$T$ static temperature

$\overline{\mathrm{T}}$ injectant to freestream static temperature ratio

$\mathrm{T}_{0}$ stagnation temperature

$\mathrm{U}$ velocity

$\bar{v}$ injectant to freestream velocity ratio

$w_{i}$ width of injection plane

$x$ streamwise, axial coordinate

$\bar{x}$ non-dimensional distance downstream of injection plane, $x / h_{i}$

y cross-stream coordinate

$\bar{y}$ non-dimensional cross-flow coordinate, $y / h_{i}$

$z$ vertical coordinate

$\overline{\mathrm{z}}$ non-dimensional vertical coordinate, $\mathrm{z} / \mathrm{h}_{\mathrm{i}}$

$\bar{z}_{j}$ jet lift-off height, mass flux center of helium normalized by $h_{i}$

$\alpha_{c}$ geometric compression angle of upper ramp surface 
$\alpha_{e}$ geometric expansion angle of upper ramp surface

$\Gamma$ circulation

$\delta$ boundary layer height

$\delta^{*}$ boundary layer displacement thickness

$\bar{\delta}$ non-dimensional boundary layer height, $\delta / h_{i}$

$\xi$ average depth of penetration of molecule due to diffusion

$\rho$ density

$\bar{\rho}$ injectant to freestream density ratio

$\phi$ equivalence ratio

$\omega$ vorticity vector

\section{Introduction}

This investigation was motivated by current efforts to develop a single-stage-to-orbit hypersonic vehicle. Through much of the flight envelope, the aircraft will be propelled by supersonic combustion ramjet (scramjet) engines relying on hydrogen-air reaction. Supersonic combustor flow is dictated by the high static temperature rise and significant total pressure loss which would be coincident with a further deceleration of the flow. Thus, for typical operating conditions, flow speeds between 6000 and $15000 \mathrm{ft} / \mathrm{s}$ will be realized, and the time-scale required for complete combustion of the oxidizer and fuel will be on the order of $1 \mathrm{~ms}$. The problem of providing rapid molecular-scale mixing in this system is compounded by poor shear-induced mixing characteristic of high Mach number shear layers, and a requirement that a significant component of the momentum of the fuel be directed parallel to the intended thrust vector to derive as much thrust from the system as possible. Further, vehicle performance in general, is closely tied to the efficacy of the combustion process. Kumar, Bushnell, and Hussaini ${ }^{1}$ have outlined additional difficulties associated with enhancing this mixing-rate dominated combustion process.

\section{Shock-Enhanced Mixing}

Implementation of shock-enhancement in a realistic scramjet injection scheme was preceded by extensive efforts at the California Institute of Technology to study two-dimensional, unsteady shock interaction with circular gas inhomogeneities. Haas and Sturtevant ${ }^{2}$ noted that " ... interaction of shock waves with fluid nonuniformities modifies the geometry and amplitude of the wave fronts by reflection, refraction, diffraction, vorticity and entropy production, and transport." In light of vorticity production, these phenomena will be generalized as shock-generated vorticity. The predominant production mechanism is that of baroclinic torque. Consideration of the baroclinic source term in the vorticity equation,

$$
\rho \frac{\mathrm{D}}{\mathrm{Dt}}\left(\frac{\stackrel{\omega}{\sim}}{\rho}\right)=\frac{1}{\rho^{2}} \nabla_{\rho} \times \nabla_{p},
$$

shows that vorticity is generated in a flow field when pressure gradients and density gradients are non-parallel. For the case of shock passage over a circle of light gas imbedded in air, imposition of the pressure gradient associated with the shock, on the density gradient at the interface between the air and the light gas inhomogeneity, results in deposition of vorticity along the density gradient. The vorticity generates large-scale convective mixing and coalesces into a counter-rotating vortex pair. For a light gas inhomogeneity, the sense of the vortex pair is such that it migrates in the direction of shock motion. Further studies by Yang $^{3}$ have elucidated the effects of shock strength and the scale and nature of the density gradient on the development of the flow field. Yang has also presented studies of multiple density inhomogeneities of various geometries and orientations.

Marble, Hendricks and Zukoski ${ }^{4}$ suggested the above as a canonical model for the case of the passage of a cylindrical jet of hydrogen fuel through an oblique shock wave. As the column of light gas passes through the pressure gradient associated with a weak oblique shock, streamwise vorticity will be deposited on the fuel air interface. The vorticity will then act to modify the surface of the jet. Large-scale stirring of the flow field will result directly in increased molecular-scale mixing as the interfacial area between the two gases in increased and species gradient dimensions decreased. Further, Marble et al. ${ }^{5}$ showed that the time-scale on which the mixing occurs is short enough to be of interest for combustion enhancement in scramjet applications.

\section{The Contoured Wall Fuel Injector}

The success of the two-dimensional shock-induced mixing program and demonstration of the threedimensional analogy ${ }^{4}$ encouraged the exploration of a low drag injection system in which the basic concept of shock-generated streamwise vorticity could be incorporated into a scramjet combustor. A geometry was conceived in April 1987 and presented by Marble et al. ${ }^{5}$. A diagram of the injection geometry is shown in Figure 1. The injector consists of alternate compression ramps and expansion troughs. The end of each ramp houses a nozzle discharging the injectant. Fuel is injected parallel to the intended thrust vector to provide full utilization of the momentum of the injectant. In the plane of injection, 
the flow in the channels between the injectors is turned parallel to the freestream, forming a weak oblique shock. This shock is drawn on the side view of Figure 1. The action of the shock is two-fold. First, the pressure gradient associated with the shock intersects the density gradient existing between the light fuel and the air, generating axial vorticity. Second, based on the twodimensional analogy, it was hypothesized that the vorticity would coalesce into a counter-rotating vortex pair of a sense that would cause migration of the fuel away from the wall. Thus, it was hoped that shockimpingement would act to enhance both mixing and jet penetration.
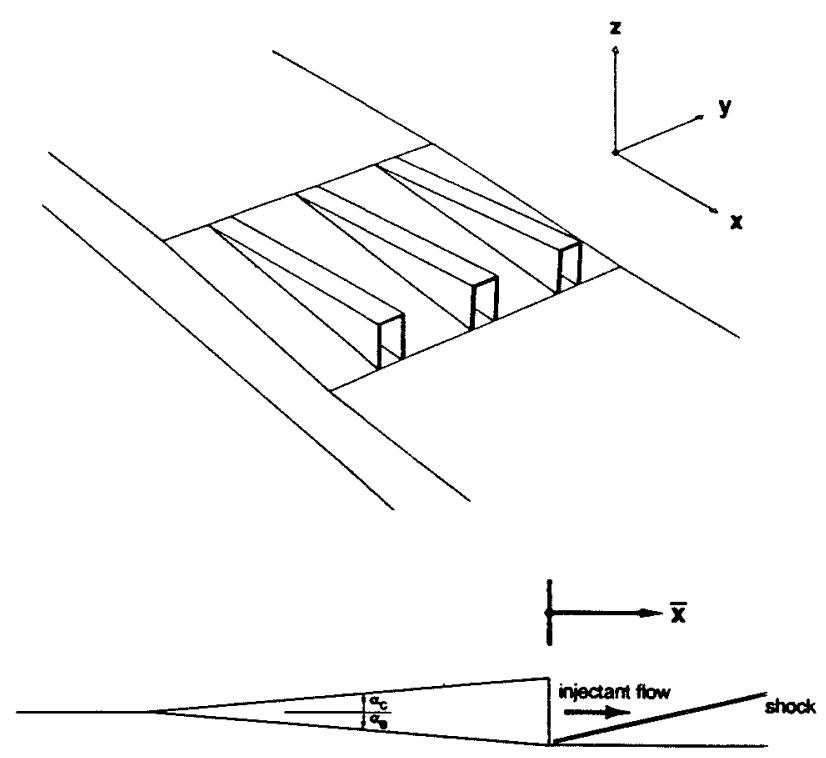

SIDE VEW

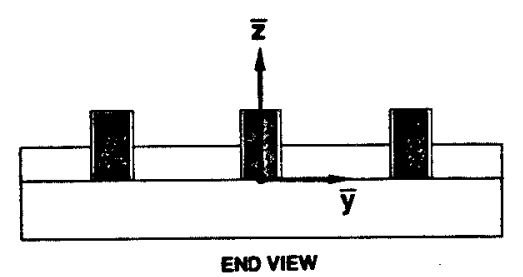

Figure 1. Diagram of contoured wall injectors and definition of axes.

Axes and pertinent geometrical parameters are detailed on the drawing of Figure 1. The barred quantities $\bar{x}, \bar{y}$, and $\bar{z}$ ) denote distances normalized by the height of the injection plane, $h_{i}$. All of the injectors studied had $2 \times 1$ rectangular injection planes $\left(h_{i}=2 w_{i}\right)$ and were characterized by the expansion and compression angles of the ramps ( $\alpha_{e}$ and $\alpha_{c}$ ), and the spacing between the injectors.

\section{Scope of the Investigation}

The results presented here are part of a broad study of a class of contoured wall fuel injectors presented in reference 6 . The goal of the study was to demonstrate the application of shock-enhanced mixing in a realistic scramjet injection geometry. Helium was used to simulate hydrogen fuel. The effort involved extensive experimental wind-tunnel testing as well as a significant computational effort which both complemented and extended the experimental investigations. Several salient parametric dependencies were investigated including those associated with injector spacing, ramp geometry, wall boundary layer effects and injectant to freestream pressure and velocity ratios. Injector performance was evaluated in terms of mixing, losses, jet penetration and heating considerations.

The focus of this paper is the detailed description of flow phenomena associated with a single geometrical configuration subject to fixed injectant and freestream conditions. Results of the parametric study will be presented in a subsequent publication?

The geometry described herein was characterized by even compression and expansion of the injector ramps $\left(\alpha_{e}=\alpha_{c}=4.76^{\circ}\right)$ and array spacing such that the trough width was equal to approximately three times the width of the injection plane. The injection planes were 1.0 in. high by 0.5 in. wide. Results from an array of three injectors are presented. Freestream conditions corresponded to $M=6$, with helium injection at $M=1.7$. Injectant conditions were chosen so that the injectant to freestream pressure and velocity ratios, $\overline{\mathbf{p}}$ and $\overline{\mathbf{v}}$, were 1.0 and 1.33 , respectively. The turbulent wall boundary layer entering the injectors was one fifth the height of the injection plane $(\bar{\delta}=0.2)$.

\section{Mixing and Combustion}

Combustion requires mixing on a molecular scale. Limits of spatial and temporal resolution precluded direct measurement of molecular-scale mixing in these studies. Unless otherwise stated, referrals to mixing performance relate to spatial scales which were discernable within the experimental and computational resolution. Mixing on a molecular scale will be inferred from these results. Increase in large-scale stirring of the flow field is a kinematical precursor to strong molecularscale diffusive mixing via an increase in interfacial 
surface area and a narrowing of gradient dimensions between the two gases. Lack of temporal resolution in these studies required that phenomena associated with the time-fluctuating component of the flow field be realized largely as they were manifested in the timemean flow field. While this allowed for estimation of the scale and importance of these effects, it did not provide sufficient information for detailed analyses.

The applicability of the results of this study to scramjets is further limited since helium was used to simulate the hydrogen fuel. Yeung ${ }^{8}$ has modelled premixed hydrogen-air reactions for conditions corresponding to a variety of flight Mach numbers. For flight at Mach 15 with Mach 5 combustor flow, inlet static conditions of $T$ $=1630^{\circ} \mathrm{C}, \mathrm{p}=15 \mathrm{psia}$, and mean flow speeds of 13000 $\mathrm{ft} / \mathrm{s}$ may be typical. Yeung's calculations show that the time required for initiation of a hydrogen-air reaction for such conditions is on the order of $25 \mu \mathrm{s}$. While strongly dependent on pressure, the result allows estimation of the extent to which scramjet combustion is controlled by mixing. For an injector geometry with a characteristic length scale of one inch, this reaction time corresponds to fluid travel of approximately four characteristic lengths. Thus, the reaction is not mixing-controlled in the classic sense. It is, however, mixing-rate dominated and investigation of hypervelocity mixing is germane to the reacting system.

In general then, treatment of the reacting system must include finite-rate chemical kinetics. It is not clear what role the heat release associated with the hydrogen-air reaction will play in damping/amplifying both largescale and molecular mixing. In the above simulation, a combustor end temperature of $\mathrm{T}=3100^{\circ} \mathrm{C}$ was projected, so significant heat release is expected. Any dependence of fluid dynamics on chemical forcing will be absent in the mixing studies presented.

\section{ExperimentalApparatus and Methods}

The tests were conducted in the NASA Langley High Reynolds Number Mach 6 Wind-Tunnel. The tunnel is an open-loop blowdown type facility, with a 42 in. long, 12 in. diameter circular test section. The tests were conducted at $\mathrm{p}_{0}=1000 \mathrm{psia}, \mathrm{T}_{0}=246^{\circ} \mathrm{C}$. The static properties associated with these conditions were $\mathrm{p}_{\infty}=$ 0.63 psia and $\mathrm{T}_{\infty}=-210^{\circ} \mathrm{C}$, with a freestream Reynolds number of $15 \times 10^{6} / \mathrm{ft}$. The tunnel was equipped with a three-axis traverse and a helium supply system both designed by NASA personnel for these studies.

A modular wind-tunnel model was designed to allow testing of a variety of injector configurations. A crosssection view of the injector region of the model is shown in Figure 2. The model was in the form of a "T", with the injector array built into the upper surface of the flat plate to simulate one interior wall of a scramjet combustor. The geometry discussed in this paper was identical to that of Figure 1, with three injectors spanning the plate laterally. Helium was injected through $M=1.7$, two-dimensional nozzles. One of the nozzles was instrumented for total and static pressure and total temperature measurements to determine helium plenum conditions. Air injection was provided near the leading edge of the model to vary the scale and nature of the boundary layer. For the results presented, no air was injected. The flat plates extended 15 inches upstream of the injector ramps and 21 inches downstream.

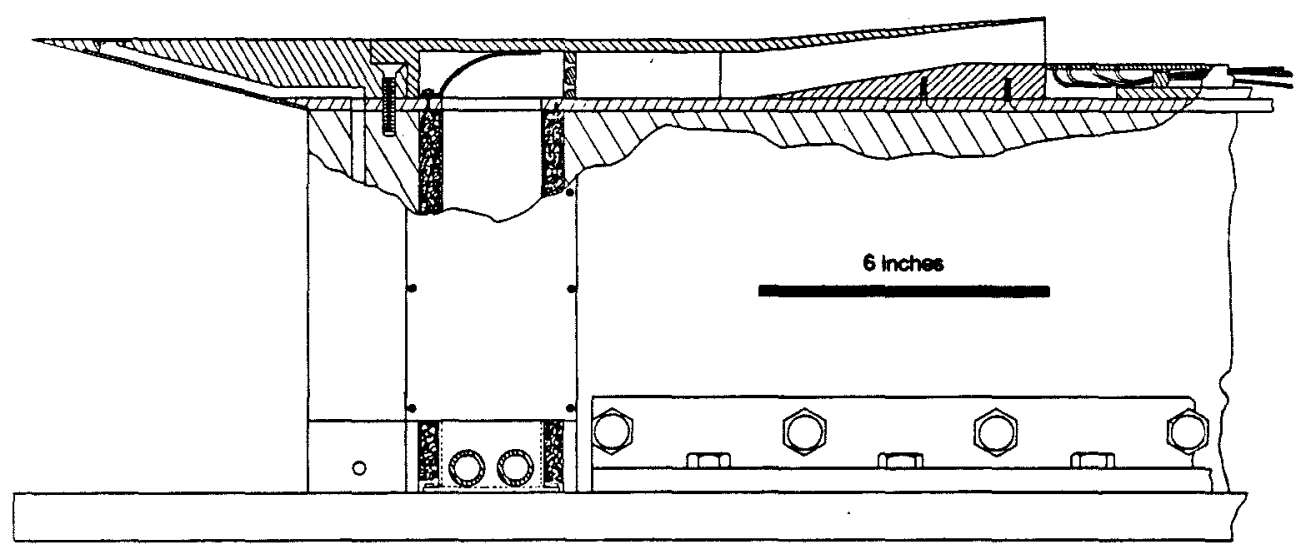

Figure 2. Cross-section view of injector region of wind-tunnel model. 
Three-dimensional flow field surveys were the primary means used to study the flow field. Four rake-mounted probes were used to investigate the flow: a cone static pressure probe, a pitot probe, a total temperature probe and a composition probe. Data collection was automated using a personal computer-based, data acquisition system.

\section{Helium Concentration Measurements}

The composition probe was based on the response of a hot-film anemometer probe in a binary gas mixture. The design and application of this device are presented in detail in reference 6 . In a helium-air gas mixture, the anemometer output, $\mathrm{E}$, is a function of pressure, temperature, velocity and composition. A sample was withdrawn from the flow field and allowed to pass over a hot-film probe. The probe was located upstream of a choked orifice such that velocity was fixed for a given pressure, temperature and composition. Further, prior to passage over the hot-film, the gas sample was thermally fixed by using a heat exchanger and in fact, locating the entire device within an ice-water bath. While removing an additional variable from the problem, thermal conditioning also enhanced sensitivity, effectively increasing the allowable overheat ratio for the hot-film anemometer. (Without cooling the sample, the high total temperatures in the flow would have allowed only very slight overheat without exceeding the operating temperature for the hot-film.) Thus, the velocity and temperature were fixed for a given pressure and composition, and $\mathrm{E}$ was dependent only on pressure and helium mass fraction. Pressure was measured at the location of the hot-film probe. An automated calibration system was developed which allowed daily calibration of device response to pressure and composition. In practice a sample was withdrawn from the flow, the pressure at the hot-film location, $p$, and the anemometer output were measured, and composition was determined from a bilinear interpolation of $E$ and $p$ in the calibration space.

A hot-film probe was used rather than the more standard hot-wire probe because of the calibration drift which results from changes in the thermal accommodation coefficient for hot-wire probes in helium-air mixtures. ${ }^{9,10}$ Care was taken to assure that sufficient mass flow was sustained through the probe so that the bow shock that formed ahead of the sampling tip was swallowed. The sampling tip was designed with an internal expansion to make this possible. If the shock is not swallowed, the strong pressure gradients existing between the shock and the probe tip cause preferential deflection of light gas species around the probe so that the sample is enriched with heavy gas species. Separation of gas species in this manner can be nearly complete $^{11,12,13}$ and proper design and mass flow monitoring are imperative for isokinetic sampling.

In final form, the gas sampling apparatus allowed determination of helium mole fraction with less than $\pm 3 \%$ error throughout the entire operating envelope ( $M=$ 6 air at $\mathrm{p}_{0}=1000 \mathrm{psia}, \mathrm{T}_{0}=246^{\circ} \mathrm{C}$, to $\mathrm{M}=1.7$ helium at $\mathrm{p}_{0}=3.4 \mathrm{psia}, \mathrm{T}_{0}=-50^{\circ} \mathrm{C}$ ). Response time on the order of 1.5 seconds, combined with wind-tunnel run times of greater than one hour allow detailed surveying of the injector flow field.

\section{Flow Visualization}

A collaborative effort was undertaken with personnel from the Optical Spectroscopy Section of NASA Langley Research Center's Instrument Research Division to investigate the application of planar Rayleigh scattering in the High Reynolds Number Mach 6 WindTunnel. All issues associated with the application of this technique as a diagnostic measure were resolved by NASA personnel (particularly Drs. Shirinzadeh, Balla, Hillard, Anders and Exton). Interpretation of the results in terms of fluid dynamics was conducted jointly. Difficult conditions in the wind-tunnel precluded use of the technique as a quantitative tool. In fact, even qualitative evaluations were hindered by various complicating factors, including molecular clustering and possible solidification of some species. The technique provided temporally-resolved planar images which, in light of comparisons with the time-mean flow survey data, allowed insight into the flow which was vital in estimating the scale and importance of unsteady phenomena. A detailed presentation of the apparatus, sampling procedures, post-processing methods, and limiting factors has been presented by Shirinzadeh et al. ${ }^{14}$ Discussions of the influence of molecular clustering on Rayleigh measurements in a similar flow field are contained in references 15 and 16 .

\section{Computational Methodology}

The difficulties associated with the experimental investigation of the contoured wall fuel injectors made application of numerical modelling an attractive complement to the experimental effort. For these purposes the SPARK3D code ${ }^{17}$ was obtained from the Computational Methods Branch at NASA Langley Research Center. The code allowed solution of the three-dimensional Navier-Stokes, energy and species continuity equations for a multiple species system undergoing chemical reaction. The code has been used by several investigators to predict reacting and non- 
reacting flow about other scramjet injector geometries. ${ }^{18-21}$ For this study, the code was used in a non-reacting mode with $M=1.7$ helium injected into $M$ = 6 air to allow direct comparison with the experiments. The system of equations was advanced in time until convergence at steady state was obtained. Typical domains consisted of 266,000 grid points.

First order boundary conditions were applied to simulate an infinite array of injectors. Viscous conditions were applied on the walls of the injector. Open boundary treatment was used on the top of the domain. A twolayer, algebraic, eddy-viscosity turbulence model was included with the code. The ability of this model to accurately represent the complex mixing phenomena associated with the contoured wall fuel injectors was strongly questioned. Application of an inappropriate turbulence model can influence the global solution in a manner which is difficult to resolve. As a result, a decision was made to limit the scope of the computations to a laminar simulation. While global phenomena were compared directly with the experiments, mixing rates were compared only qualitatively.

\section{Results}

Figure 3. contains a temporally-resolved (30ns pulse duration) shadowgraph of the flow field about the injector array. Flow is left to right. The density variations in the flow field were highly threedimensional and the locations of their signatures in the photograph correspond to a line-of-sight integration across the three injectors in the array. Both the shock generated by the beginning of the injector ramps and that due to turning of the flow in the troughs between the ramps at the exit plane are apparent. The latter of these was responsible for baroclinic vorticity generation through interaction with the density gradients associated with the injectant/air interfaces. In fact, passage of the helium jet (represented by the two parallel white lines which intersect the right edge of the photograph nearly perpendicularly) between the shocks formed in the troughs is topical evidence that the contoured wall geometry provided shock-enhancement as envisioned. Note the strong separation between the lower boundary of the jet and the wall.

The shock generated by the compression of the upper ramp surface foretells a second vorticity generation mechanism associated with the ramps. High pressure existed above the ramps with lower pressure fluid in between. The pressure imbalance generated flow from the upper surface of the ramps into the troughs causing the fluid to roll up along the corner of the ramps. This will be termed 'ramp-generated vorticity'.

Thus, the mechanisms for two sources of vorticity are evidenced in the photograph. The sense of the vorticity which resulted from these two mechanisms was the same. Computational studies ${ }^{6}$ showed that while the strength of the vorticity from these two sources was similar, that associated with shock-enhancement was more effective in mixing and providing strong jet penetration. The may be a result of its location in the flowfield with respect to the injectant/air interface. Shock-impingement assures seeding of vorticity directly on the light/heavy gas interface where mixing is desired. The ramp-generated vorticity was formed remote from the mixing interface.

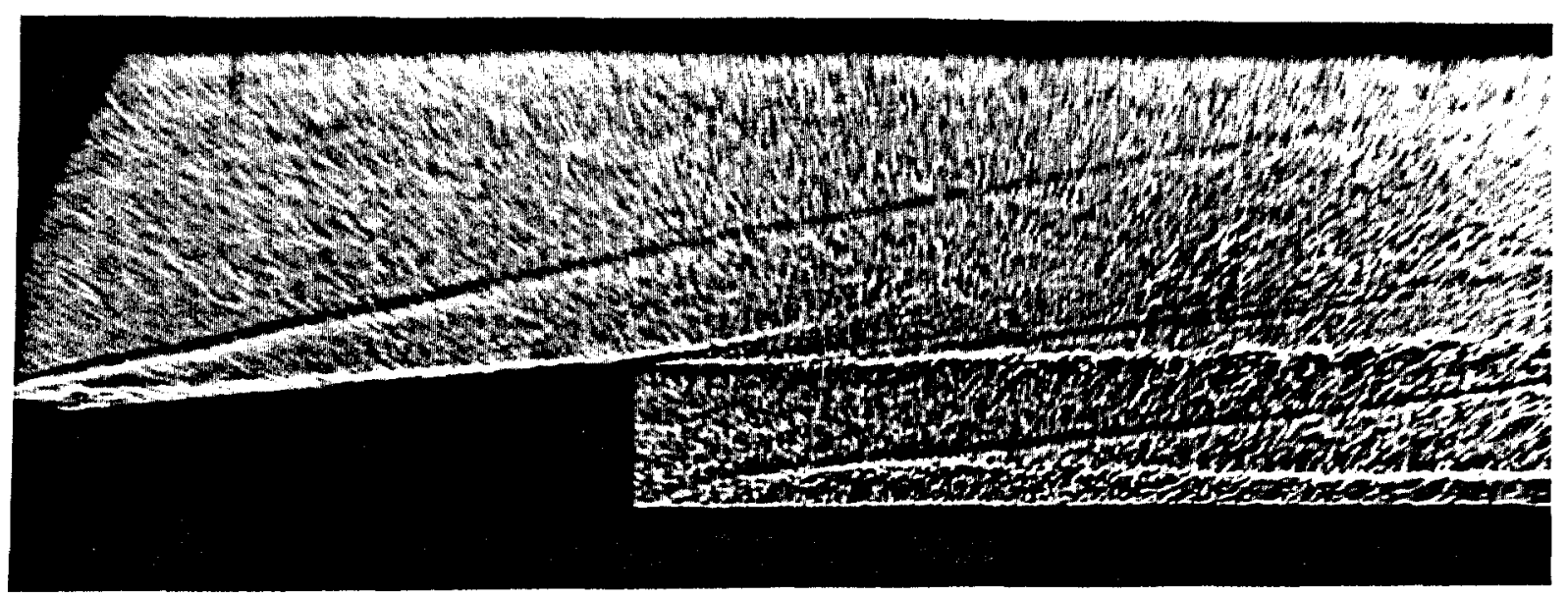

Figure 3. Shadowgraph of injector flow field. 


\section{Flowfield survey}

The dynamics of the helium jet are displayed in more detail in Figure 4. Contours of constant helium mass fraction are shown for various axial stations downstream of the injection plane, $(\bar{x}=0,1,4,8$, and 13). The data were obtained using the composition probe described above. The data have been mirrored about the plane of symmetry at $\bar{y}=0$. The edges of the injector are at $\bar{y} \pm$ 0.25. The edges of each of the plots correspond to the planes of symmetry between the injectors in the array. The maximum helium mass fraction is tabulated above each of the plots. It must be stressed that these data are time-mean and the manifestation of the unsteady component of the flow in these plots has not been addressed. Also, in these plots and others shown, subgrid scale features should not be interpreted. The experimental sampling grid has been overlaid on one of the plots of Figure 4.

The strong lift-off of the jet apparent in the shadowgraph is clear in these data as well. Complete separation of the jet from the surface occurred at approximately one injector height downstream of injection. The formation of a strong counter-rotating vortex pair in the helium jet is evidenced by the form of the helium signatures. The sense of the vorticity is such that it caused the pair to migrate from the wall into the freestream.

More details of the flow about the ramps are apparent in the pitot pressure surveys shown in Figure 5. At $\bar{x}=0$ high pressure existed above the ramps as a result of compression of the upper ramp surface, and low pressure between, due to expansion in the troughs. This pressure gradient produced the ramp-generated vorticity discussed above.

The nature and importance of the boundary layer is also shown in these plots. Because of the above pressure gradient, the boundary layer was drawn from the compression surfaces and into the troughs between the ramps. Due to the strong displacement effect of the hypersonic boundary layer, it acted to modify the effective wall geometry from the perspective of the flow external to the boundary layer. Consideration of the pitot pressure contours in the injection plane, shows that the form of the boundary layer was such that it did not inhibit shock formation in the vicinity of the helium/air density gradients. Counter to expectations, a tongue of high momentum flow was maintained in the corner of the troughs, with the low momentum boundary layer fluid collecting in the center of the passageway. This allowed shock formation precisely where it was required to produce baroclinic torque: the region of helium/air density gradients. The collection of boundary layer fluid in the center of the channel between the injectors was a result of positive interaction between the geometry and the boundary flow. This positive interaction was not present for all injector spacings and boundary layer thicknesses ${ }^{7}$.

\section{Numerical simulation}

For comparison purposes the numerical data has been plotted in the same form, scale and axial locations as the experimental data plots. The helium mass fraction and pitot pressure are shown in Figures 6 and 7. The spacing between the injectors for the computational case was slightly different from that of the experimental case and the plots extend to $\bar{y} \pm 1.0$ rather than $\bar{y} \pm 1.125$. Again, these bounds represent the planes of symmetry between each of the injectors in the array. The edges of the injector ramp still correspond to $\bar{y} \pm 0.25$. Also, while the computations were carried out in a domain $0<\overline{\mathrm{z}}<$ 4.0 , they were cropped above $\bar{z}=2.625$ which was the maximum height measured experimentally. The spatial resolution provided by the computational grid was considerably better than that of the experiments. The grid has been overlaid on one of the plots of Figure 6 .

Since no attempt was made to accurately model the turbulent mixing phenomena, the mass fraction levels displayed in the simulation differ from those measured experimentally. However, the qualitative behavior and the location and form of the species gradients were accurately reproduced in the computations. Comparing the experimentally and numerically obtained pitot pressure contours (Figures 5 and 7, respectively), close qualitative and quantitative agreement is seen. It should be noted that the numerical investigations were conducted before the experiments. The excellent agreement between the results of the two was not the result of a posteriori 'tuning'. In fact, further adjustment particularly with respect to turbulence modelling, may allow even more confidence in the predictive capabilities of the simulations.

Figure 8. contains a perspective view of data obtained from the numerical simulation. Contours of constant helium mass fraction are shown at various planes downstream of the injector. The helium signatures produced by jet interaction with the counter-rotating vortex pair are reminiscent of those seen for the canonical problem of two-dimensional, unsteady, shock impingement on a circular light gas inhomogeneity. ${ }^{2-5}$ The analogy between this and the current problem is limited by other significant sources for vorticity in the flow field (ramp-generated vorticity, boundary layer, and shear), and three-dimensional effects. 

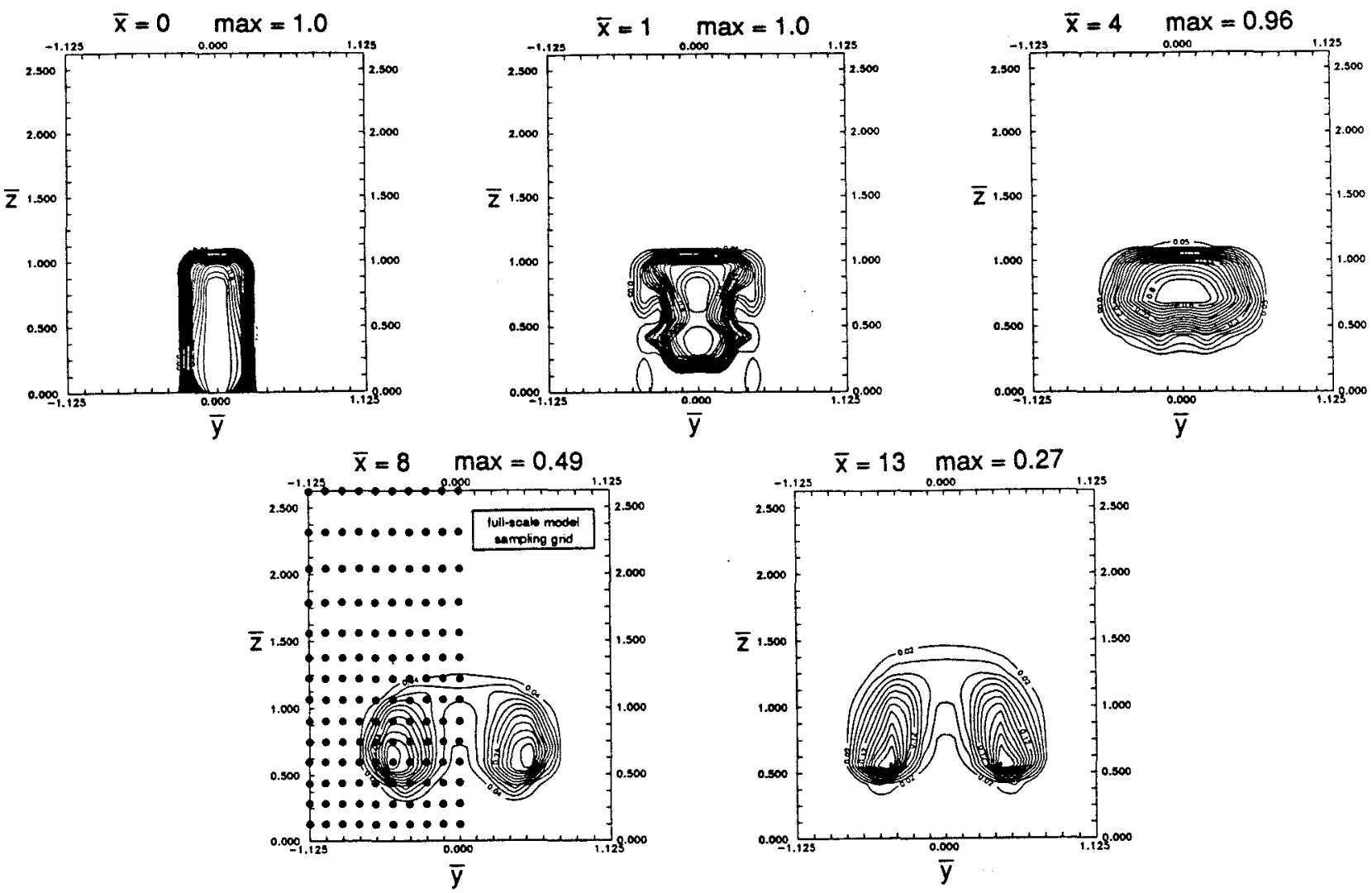

Figure 4. Contours of constant helium mass fraction, [experimental].
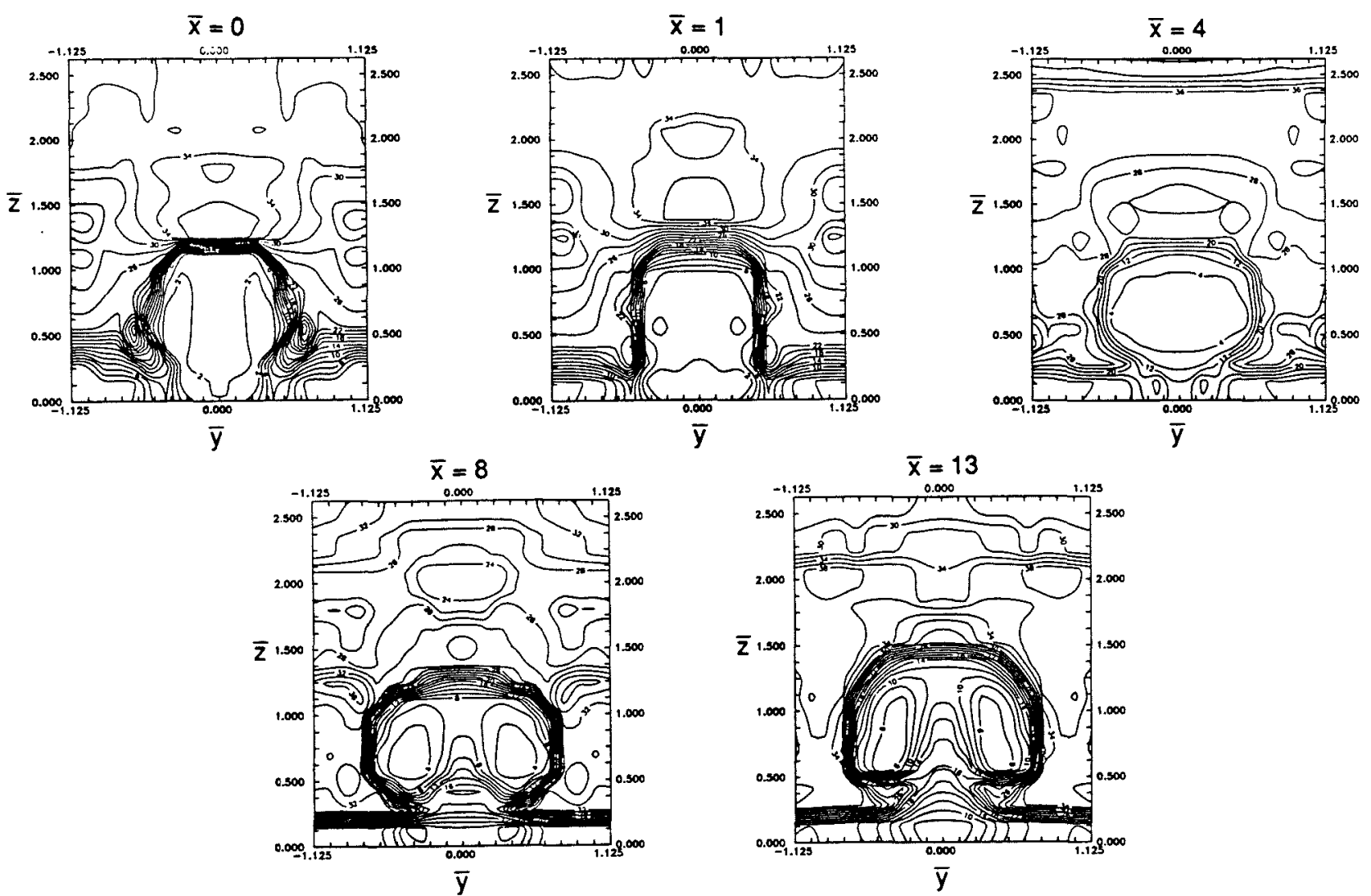

Figure 5. Contours of constant pitot pressure (psia), [experimental]. 

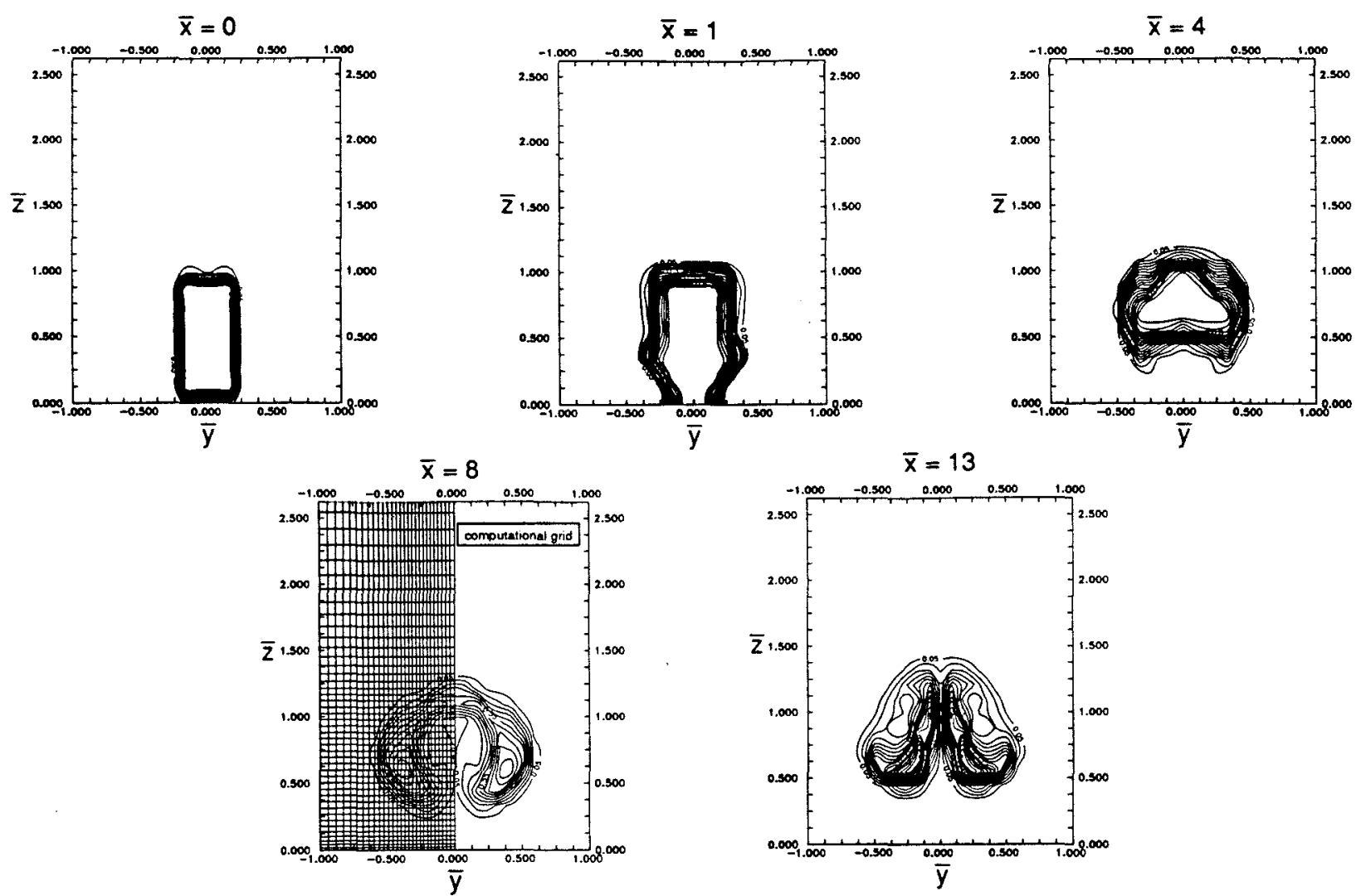

Figure 6. Contours of constant helium mass fraction, [computational].
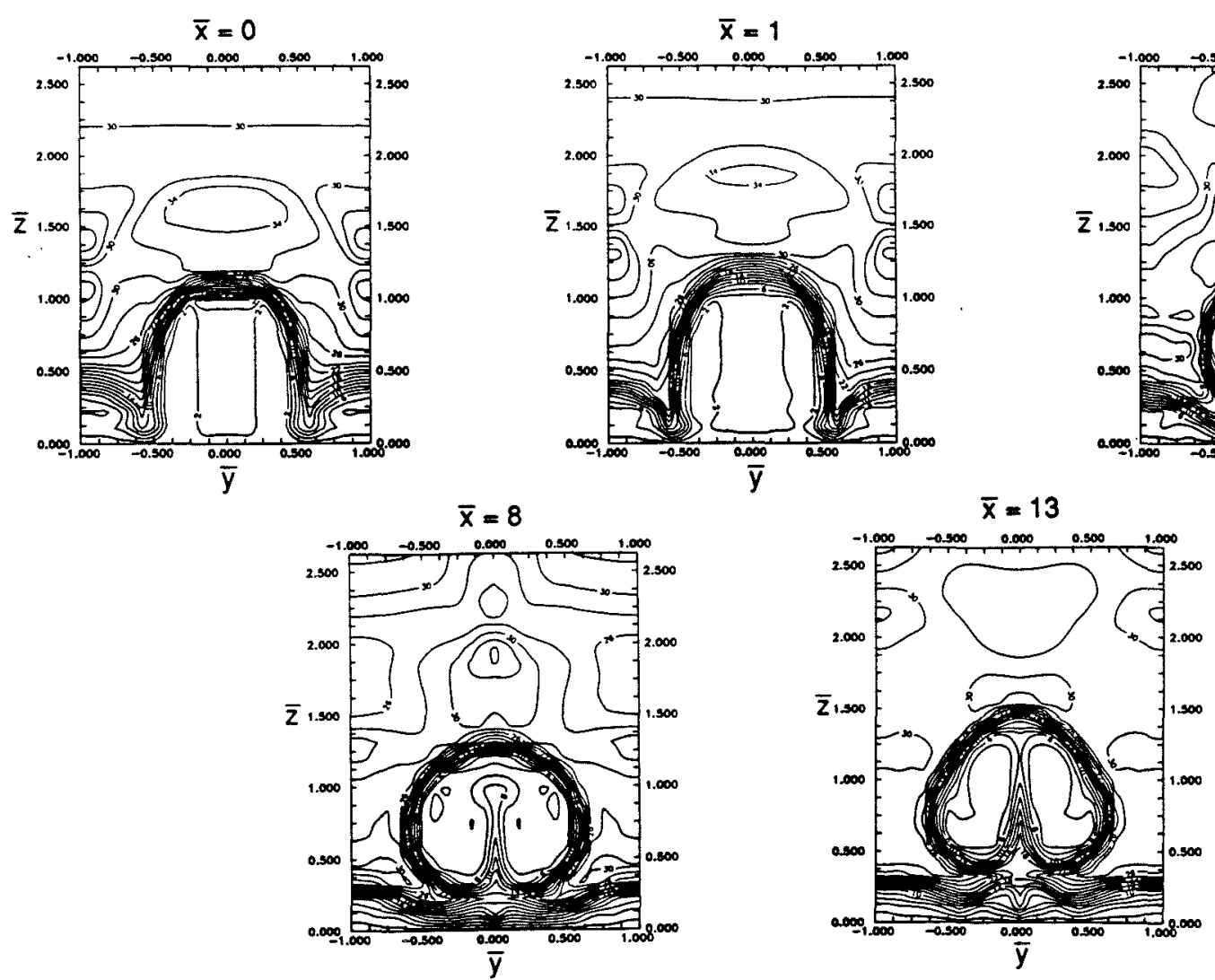

Figure 7. Contours of constant pitot pressure (psia), [computational]. 


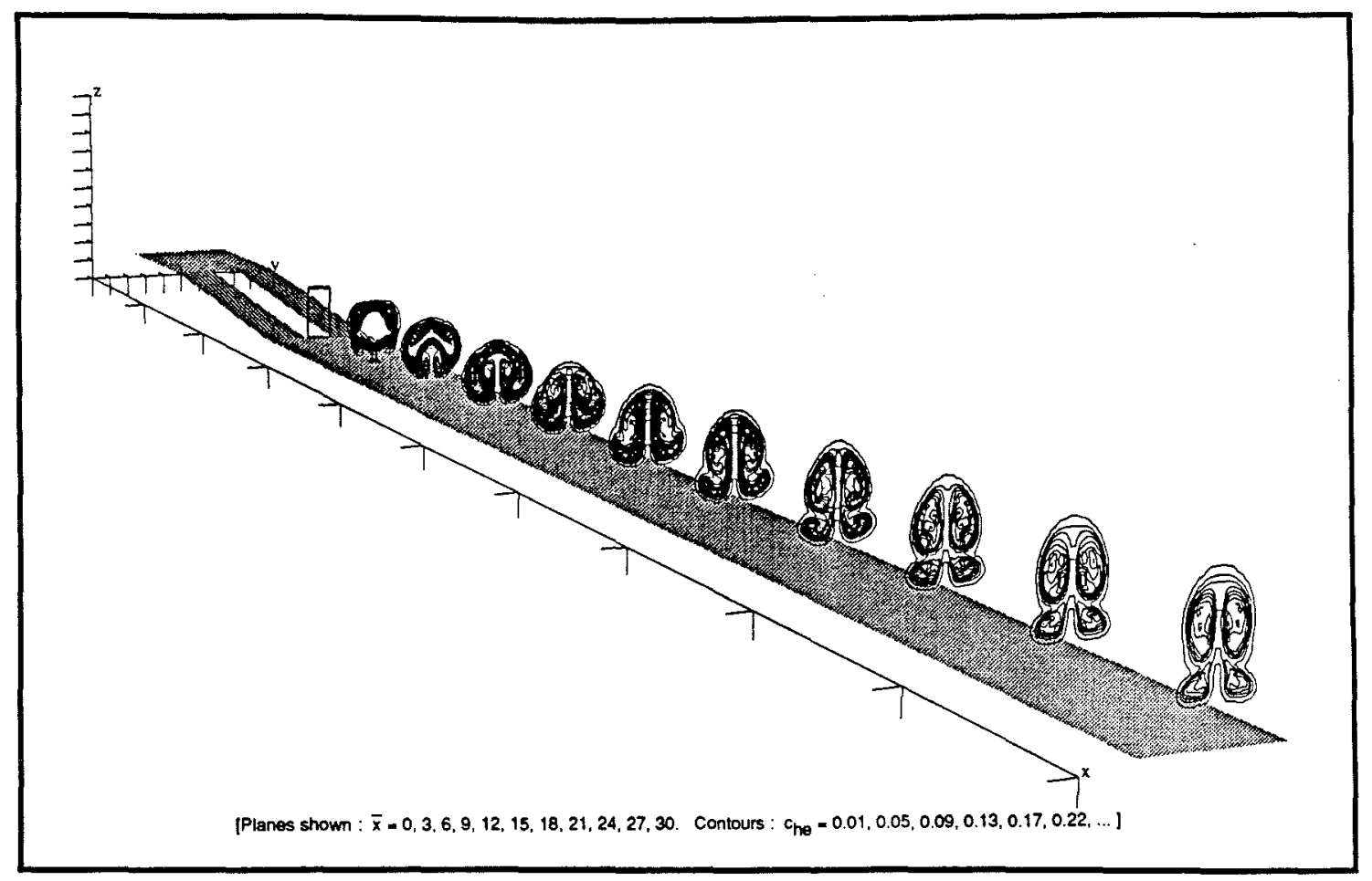

Figure 8. Perspective view of jet development. Contours of constant helium mass fraction, [computational].

\section{Thoughts on sub-grid scale structure}

The representation of the helium jet in both the experimental and computational contour plots suffers from two strong influences: temporal averaging and spatial averaging. The influence of spatial averaging on the form of the helium signatures can be estimated by considering the dynamics of the jet with respect to diffusion and convection.

Convective mixing is demonstrated by the interaction of particle traces from neighboring points in the flow field. Two examples of such interactions are shown in Figures 9 and 10 . The entrainment of air into the helium jet is displayed in Figure 9 where a fluid particle in the air is swept into the mixing helium jet. The strength of the vortical flow is shown in Figure 10. The particle path shown with the solid line makes three complete orbits about the vortex core by $\bar{x}=30$. Notably, the two helium particles, located one just above the other, take considerably different paths through the domain with the lower particle exhibiting an additional orbit. Fluid interfaces are stretched and injectant/freestream gradient dimensions are narrowed.

The relation of the strong convection displayed in these figures to the diffusive mixing was estimated by considering the growth of helium/air diffusion gradients in a steady system. The average depth of penetration of a molecule in a species gradient, $\xi$, may be approximated ${ }^{22}$ as:

$$
\xi=\sqrt{2 \mathrm{Dt}}
$$

The diffusion coefficient for helium and air at $-170^{\circ} \mathrm{C}$ and 0.63 psia was estimated using the Chapman-Enskog theory ${ }^{23}$ to be $\mathrm{D}=3.75 \times 10^{-3} \mathrm{ft}^{2} / \mathrm{s}$. At flow speeds of $3000 \mathrm{ft} / \mathrm{s}$, a particle resides between the injection plane and the end of the computational domain for $1 \times 10^{-3}$ seconds. Thus $\xi=0.033$ in. or $3.3 \%$ of $h_{i}$. Based on this, it is apparent that much of the mixing region may be in the form of tightly rolled spiral species gradients which have not yet merged due to diffusion. This signature would be hidden by artificial viscosity and spatial discretization in the computations, and by the spatial averaging of the probes and limited spatial resolution of the sampling grid in the experiments. Determination of the extent to which these layers merge and actually take on the form displayed in the experimental and numerical data was not possible, though the small diffusive length scales suggest that at least part of the flow field contains tightly layered species gradients. The extent to which these fine mixing scales may be dominated by unsteady effects is addressed below. 


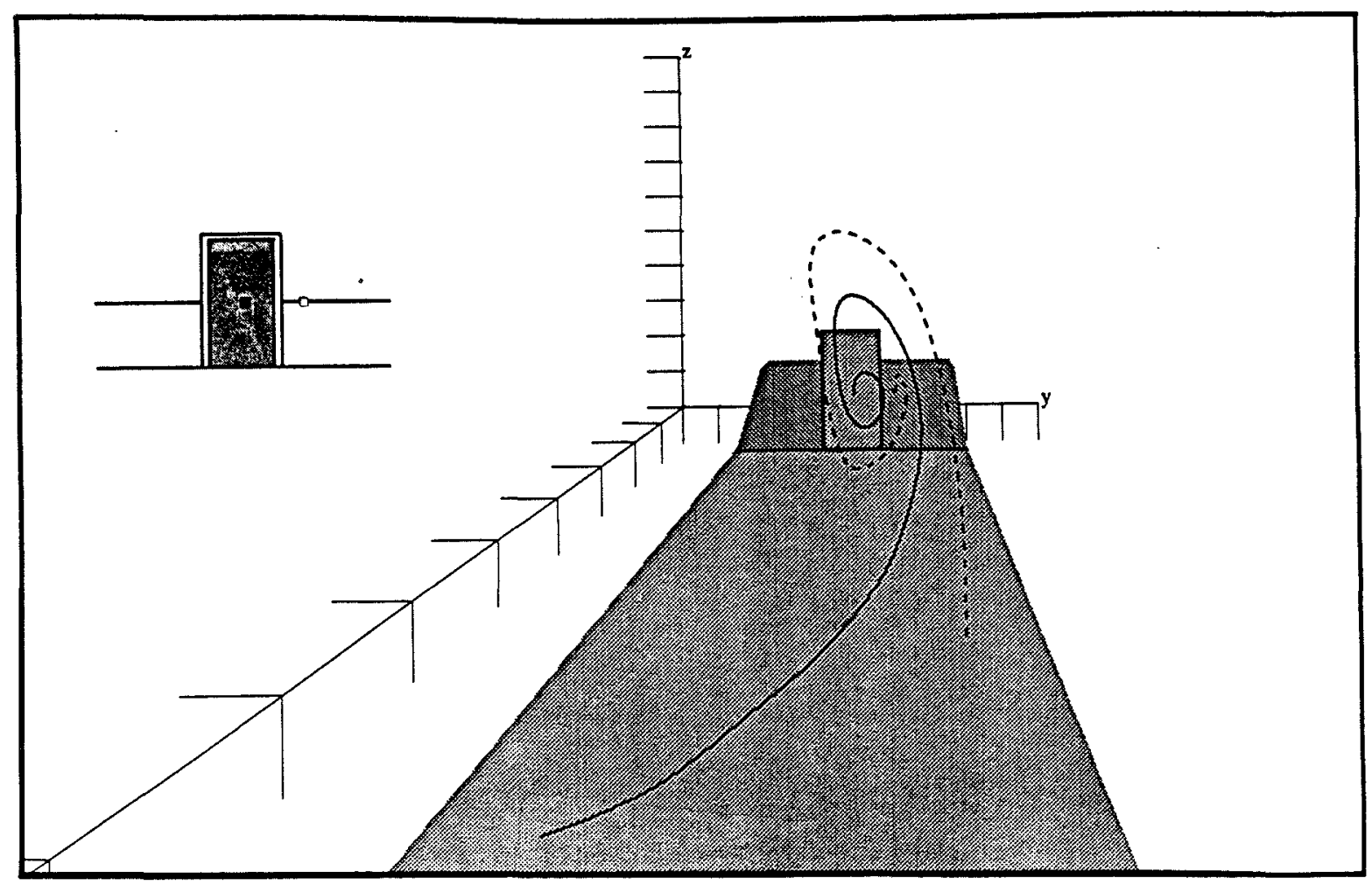

Figure 9. Particle traces \#1.

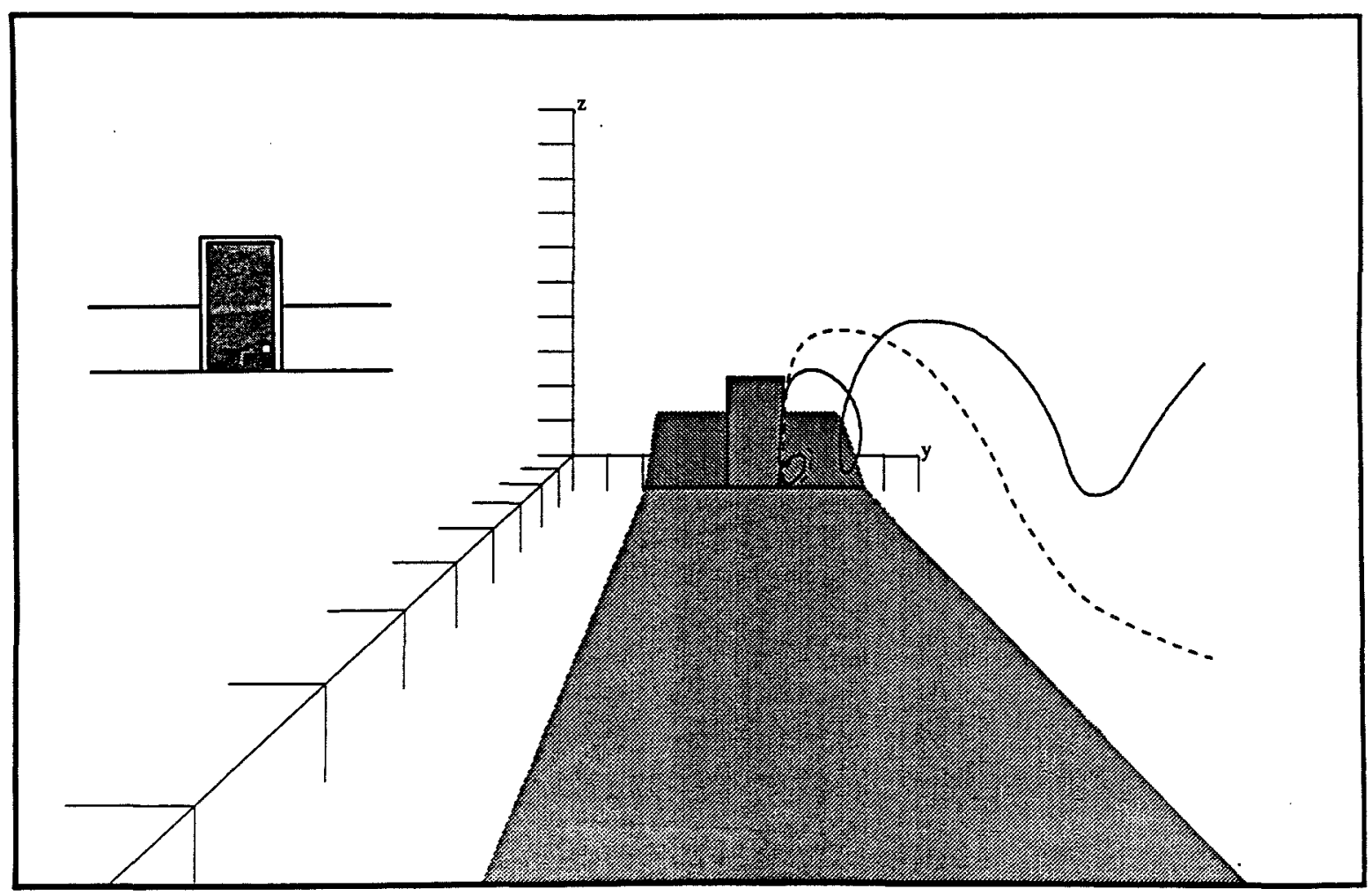

Figure 10. Particle traces \#2. 
The time-fluctuating flow

Temporally-resolved (15ns pulse duration), planar Rayleigh scattering was employed to impart a qualitative knowledge of the level and importance of the timefluctuating component of the flow field. Prior to presenting the time-resolved data the limitations of the imaging technique will be addressed through a comparison with the time-mean, rake survey data.

Four plots are shown in Figure 11. Figure 11b. is an average of 22 time-resolved, planar Rayleigh images taken at $\bar{x}=10$. Included in the figure are contour plots of static pressure, static temperature (both obtained from the raw data through the reduction technique described in Reference 6), and helium mass fraction. Due to limitations associated with molecular clustering, the optical technique was applied at $\mathrm{T}_{0}=246{ }^{\circ} \mathrm{C}, \mathrm{p}_{0}=600$ psia resulting in a Reynolds number of $9 \times 10^{6} / \mathrm{ft}$. While the other tests presented were conducted at $\operatorname{Re}=15 \mathrm{x}$ $10^{6} / \mathrm{ft}$., the rake survey data shown in Figure 10 were obtained at $\mathrm{Re}=10.5 \times 10^{6} / \mathrm{ft}\left(\mathrm{T}_{0}=246{ }^{\circ} \mathrm{C}, \mathrm{P}_{0}=700\right.$ psia) to allow closer comparison with the Rayleigh data.

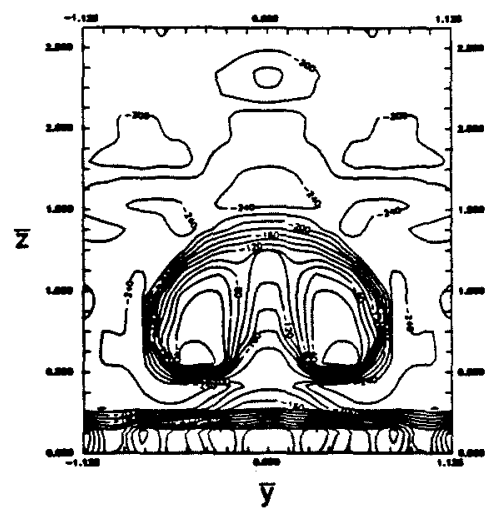

a. Static temperature $\left({ }^{\circ} \mathrm{C}\right)$

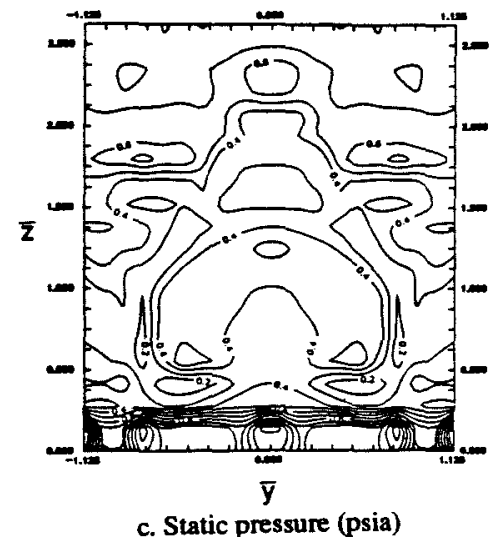

The data (in terms of deduced pressure in units of torr) are scaled linearly between zero and the maximum signal level in the image. As the scattering cross-section for helium is much less than that for the air, lower signal levels are expected in regions containing significant concentrations of helium. Lower signal levels are also noted in low pressure or high temperature regions of the air. Some regions of the flow field (e.g., the core of the helium jet and the base of the boundary layer) were below the sensing threshold of the instrumentation. White bands have been added to the gray scale to aid in identification of intensity gradients in the images. The dynamic range and spatial resolution of the Rayleigh measurements did not allow analysis of the fine-scale structure within the jet. Cursory analysis of these plots shows that to first order, variations in intensity in the Rayleigh images correspond to variations in static temperature in the flow field. This is due in part to the strong dependence of molecular clustering on local temperature. The representation of the location of gradients in helium mass fraction is accurate only in regions where the species gradients were coincident with pressure and/or temperature gradients. Thus the utility of the method for tracking the mixing interface is strongly limited.

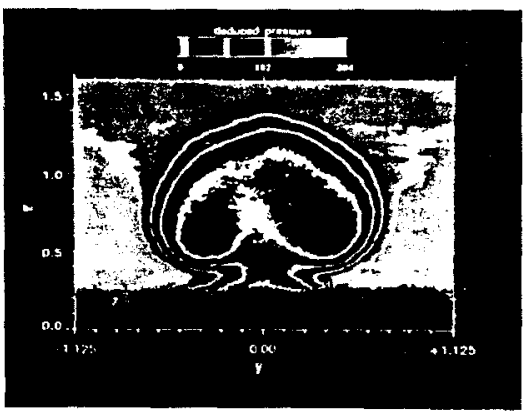

b. Mean of 22 Rayleigh images

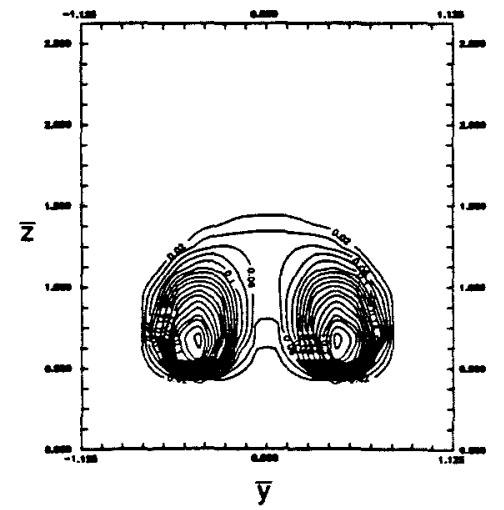

d. Helium mass fraction

Figure 11. Comparison of mean survey data with mean of 22 planar Rayleigh images at $\bar{x}=10$. 
The above limitations must be considered in interpreting the time-resolved data which are presented in Figure 12. The frames were taken 0.1 seconds apart. In 0.1 s a fluid particle moved approximately $300 \mathrm{ft}$., so the images are considered random in time. On the sides and top edges of the jet, it is assumed that lines of constant image intensity correspond to a lines of roughly constant helium mass fraction. Below the jet, a similar conclusion is not allowed, as pressure and temperature gradients were not coincident with species gradients as described above. In particular, gas sampling measurements show the stem which appears below the jet did not contain any helium (see Figure 11d). It was merely warmer, lower pressure air entrained in part from the boundary layer. The relative effects of number density variations and thermodynamics (molecular clustering) in producing these variations can not be separated. The maximum peak-to-peak spatial amplitude of the unsteady oscillations of the intensity gradients about the mean is approximately $0.4 \mathrm{~h}_{\mathrm{i}}$.
This data was used to estimate the extent to which mixing in the fine scales was dominated by unsteady effects. Broadwell and Mungal $^{24}$ have suggested that the progression from stirred fluid to mixed fluid in simple two-dimensional and three-dimensional high Reynolds number shear flows is driven by the turbulent cascade process, as the scalar diffusion time across the Kolmogorov viscous scale is negligible compared to the cascade time. For strongly augmented mixing, this is only true in regions of the jet where shear-induced unsteadiness is significant. It is projected that in the near-field of the injectors, diffusive mixing across the fine, spiraled species gradients generated by the timemean augmentation processes, contributed significantly to the level of molecular mixing. The scale of the unsteadiness in the Rayleigh images suggests that for $\bar{x}>$ 10 turbulent mixing became progressively more important. Certainly, the strong large-scale convection displayed in the particle traces of Figures 9 and 10 was superposed on the turbulent mixing, and may have enhanced this process as well.

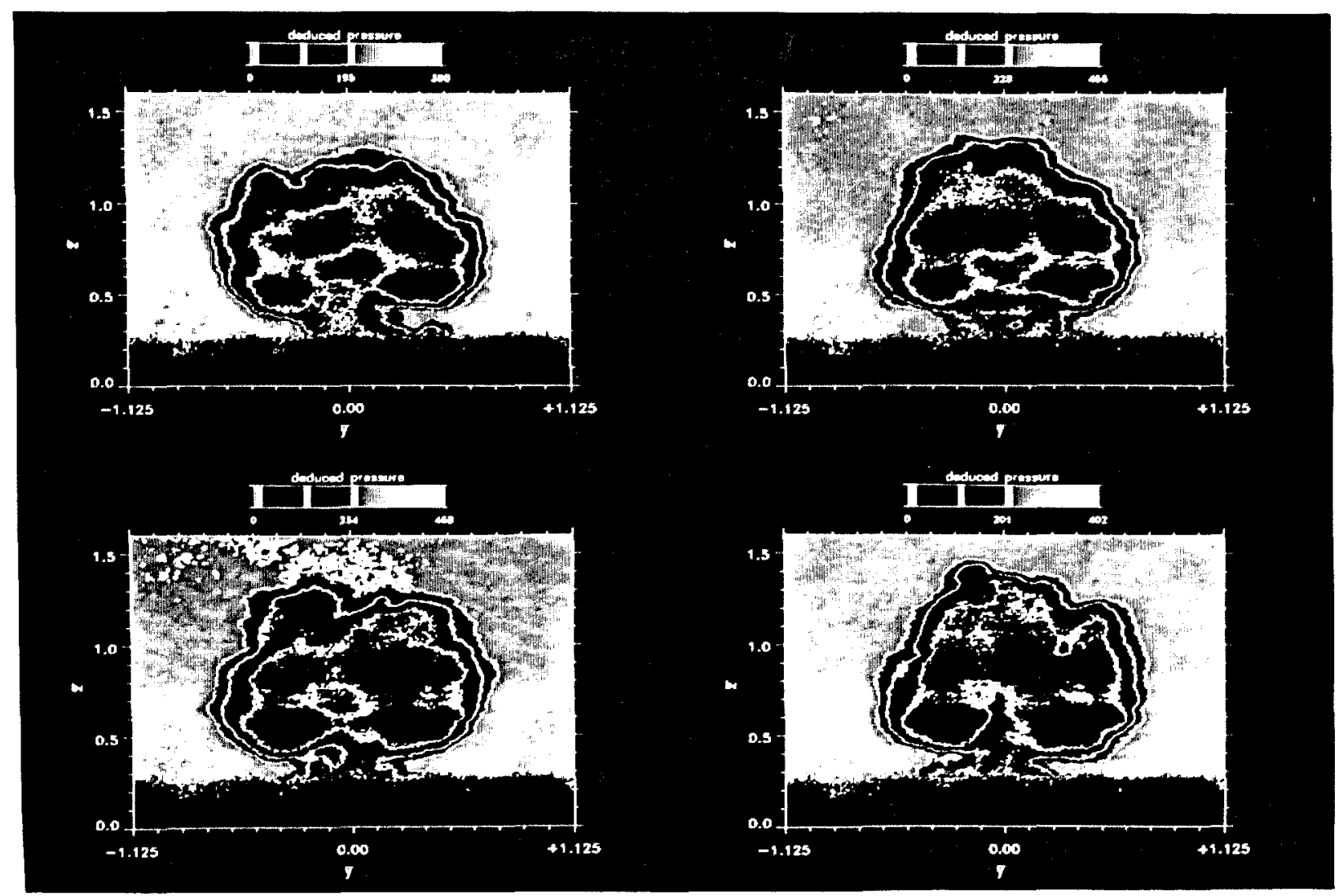

Figure 12. Instantaneous planar Rayleigh images at $\bar{x}=10$. 
Analysis of performance

The lift-off height of the jet from the surface, $\bar{z}_{j}$, is compared for the experimental and numerical data in Figure 13. This was calculated from the numerical data as the helium mass flux center, and approximated using the experimental data by calculating the helium mass fraction center. The lift-off height is a measure of the bulk behavior of the injectant downstream of the injection plane. The agreement between the experimental data and the simulation is excellent. The dip in the curve is a result of the dynamics of the helium as it was acted upon by the vorticity field. After coalescing into a counter-rotating vortex pair, migration from the wall at a constant slope of $\bar{z}_{j} \sqrt{x}=0.025$ occurred. By $\bar{x}=30$, the mass flux center was located at $\overline{\mathrm{z}}=1.3$, with the point of maximum penetration at $\overline{\mathrm{z}}=$ 2.0. Possibly more important than the bulk behavior of the jet for heating considerations, was the complete liftoff of the jet from the surface. Absence of fuel near the surface of the combustor will alleviate some of the detrimental heating envisioned in scramjet combustor applications. Impingement upon the injectant/air density gradients with the shock that was generated at the base of the jets was the factor which provided complete liftoff. $^{6}$ Notably, impingement upon the jets from above, such as with a reflected shock, acts to produce vorticity of an opposite sense. If this vorticity is dominant, a counter-rotating vortex pair will be formed of a sense that will cause an undesirable migration of the jet towards the wall.

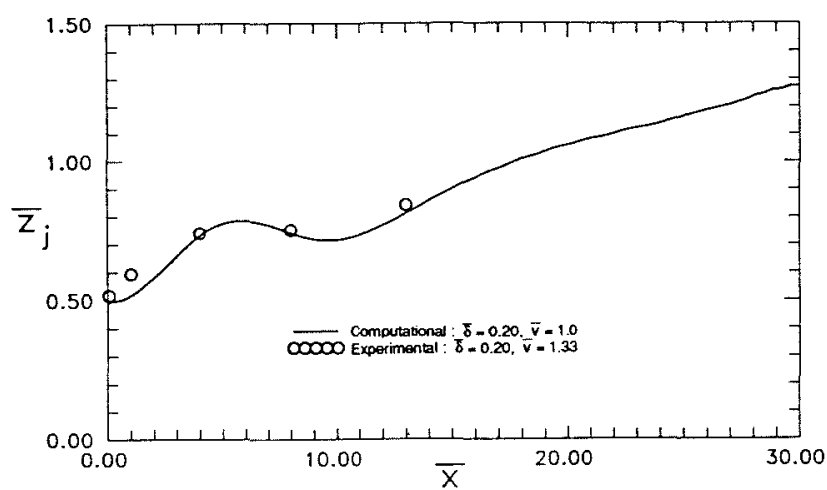

Figure 13. Lift-off height of helium jet from surface.

An integral measure of vorticity production within the flow based on the computational data is presented in Figure 14. Two strong vorticity generation mechanisms are displayed in this plot. The growth in circulation which begins at $\bar{x}=-6.0$ (which corresponds to the start of the ramps) was loosely termed ramp-generated vorticity and was a result of fluid migration from the high pressure region on the upper surface of the ramps to the low pressure region in the troughs between ramps. At $\bar{x}=0$, a large increase in circulation occurred. This increase was the result of two factors. The first of these was strong vorticity production from baroclinic torque due to shock impingement on injectant/air density gradients. This mechanism produced vorticity of the same sense as that generated by the ramp. It cannot be assumed, however, that the entire increase in circulation following injection was due to baroclinic vorticity production. A portion of the increase was due to the kinematics associated with the ramp-generated vorticity acting on the lighter injectant gas.

More detailed studies ${ }^{6}$ have shown that the shockgenerated vorticity was more effective in convectively mixing the injectant with the freestream. Shockimpingement resulted in vorticity deposition on the helium/air interface, whereas the roll-up due to the ramps occurred in the air and then propagated into the mixing region.

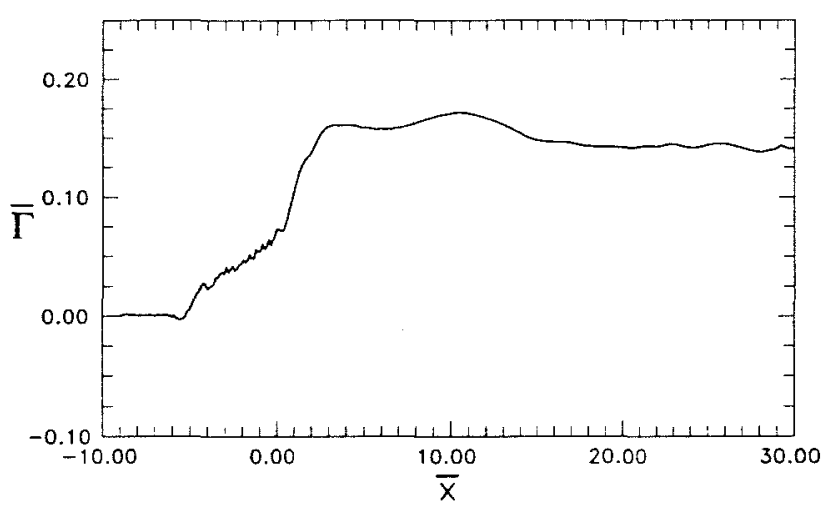

Figure 14. Circulation in y-z plane, [computational].

The peak injectant mass fraction levels in each measurement plane have been plotted for the experimental data in Figure 15. Data from a half-scale model test have been included to show the behavior at larger non-dimensional distances from the injection plane. When plotted on a linear scale (Figure 15a), a maximum decay slope of $12 \%$ mass fraction per injector height downstream occurs between $\bar{x}=5$ and $\bar{x}=10$. For $\bar{x} \geq 4$ the decay was fit with a power law proportional to $\overline{\mathrm{x}}^{-1.37}$, as shown in the $\log -\log$ plot of Figure $15 b$. 


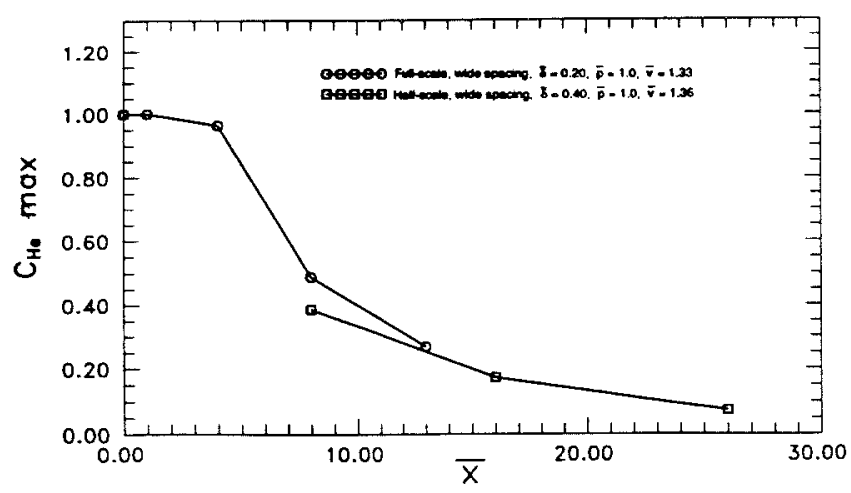

a. Linear scaling

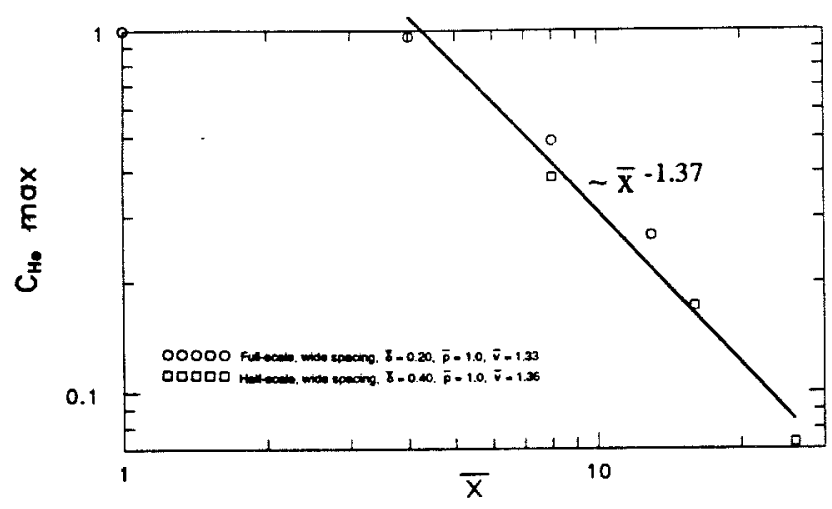

b. Log-log scaling

Figure 15. Peak helium mass fraction decay, [experimental].
More detailed mixing analyses were conducted for the numerical data. Figure 16 provides information concerning the percent of total injectant mass flux which was present in each concentration band as a function of distance downstream of the injection plane. The general characteristics of such a measure are displayed in the figure, and are highlighted by plotting both a contour map and a surface plot. Initially (at $\bar{x}=0$ ), all of the injectant existed in a pure state $\left(c_{\mathrm{He}}=1.0\right)$. This is represented by a distribution similar to a delta function. Farther downstream from the injector, more of the injected helium occurred at progressively lower mass fractions. The zero contour in the line plot, corresponds to the decay of maximum helium mass fraction plotted for the experimental results. Only qualitative comparisons between the experimental and numerical data are allowed due to the limited scope of the computational modelling with respect to mixing. Comparison of this line with the experimental data of Figure 15a shows the nature of the decay for the two cases was similar. However, the laminar mixing model produced mixing at a much slower rate. Another aspect of the mixedness measure data that is important is the percent of helium mass flux present at the lower mass fractions where the mixture ratio would promote most active burning in a hydrogen/air reaction system. By $\bar{x}$ $=30$, approximately $17 \%$ of the injected mass flux was below $5 \%$ mass fraction.

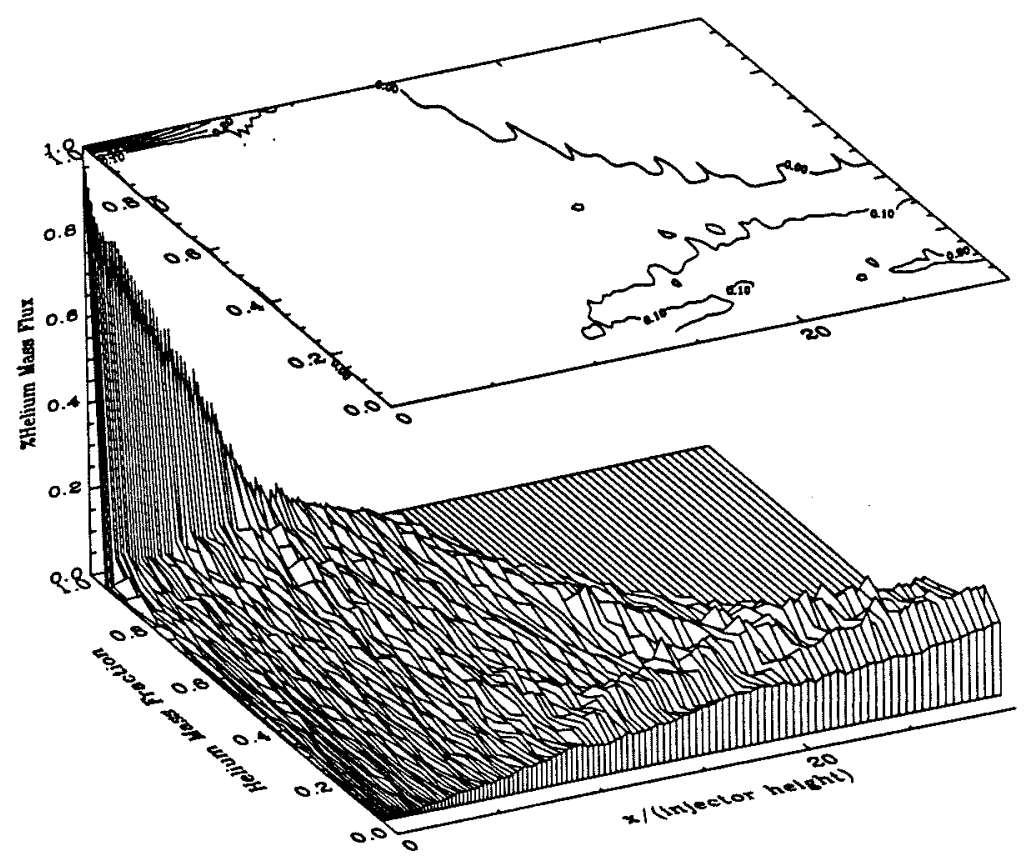

Figure 16. Mixedness measure, [computational]. 
It is important to cast jet penetration and mixing performance in light of pertinent fuel/air mass flux ratios for scramjet applications. In terms of combustor sizing, it is interesting to consider the freestream area required relative to the area of the injector exit plane if the fuel were to be homogeneously mixed to a desired mass fraction for a given set of conditions. The equivalence ratio, $\phi$, can be expressed as

$$
\phi=\frac{\overline{\dot{m}}}{\overline{\dot{m}}_{\mathrm{st}}} \text {, }
$$

where the subscript 'st' refers to stoichiometric, and the injectant to freestream mass flux ratio, $\overline{\dot{m}}$, is

$$
\overline{\dot{m}}=\frac{\rho_{i} U_{i} A_{i}}{\rho_{\infty} U_{\infty} A_{\infty}}=\bar{\rho} \bar{v} \bar{A} \text {, }
$$

or

$$
\overline{\mathrm{m}}=\frac{\overline{\mathrm{p}} \overline{\mathrm{m}} \overline{\mathrm{v}} \overline{\mathrm{A}}}{\overline{\mathrm{T}}} \text {, }
$$

where $\bar{m}$ is the injectant to freestream molecular weight ratio. Then the area ratio required to provide a desired equivalence ratio for a given set of injectant and freestream conditions is

$$
\overline{\mathrm{A}}=\frac{\phi \overline{\mathrm{m}}_{\mathrm{st}} \overline{\mathrm{T}}}{\overline{\mathrm{p}} \overline{\mathrm{m}} \overline{\mathrm{v}}}
$$

An estimation of each of these parameters was made for typical scramjet operating conditions. For $\phi=1.0, \overline{\dot{m}}_{\text {st }}$ $=0.03, \bar{m}=2 / 29, \bar{p}=1.0, \bar{v}=1.0$, and $\bar{T}=1 / 6$, then $\bar{A}=$ 0.07 . So the injectant must be mixed into an area approximately 15 times the area of the injection plane. If this area is fixed laterally by the planes of symmetry separating each of the jets in the array, then the vertical extent of the area would be approximately $\bar{z}=4.0$ for the wide spacing configuration. For the freestream velocities of these tests, penetration on that order would be expected by $\bar{x} \approx 60$. For the specific conditions of this study with $\phi=1.0, \overline{\mathrm{m}}_{\mathrm{st}}=0.03, m=4 / 29, \overline{\mathrm{p}}=1.0, \overline{\mathrm{v}}$ $=1.33$, and $\bar{T}=2.5$, the area ratio is much higher with $\overline{\mathrm{A}}$ $=0.41$. The high static temperature ratio is only partially offset by the increased molecular weight of the injectant. Considerably less air would be required to be entrained by the helium jet.

\section{Conclusions}

An investigation of a contoured wall fuel injector has been presented. The injector design was aimed at enabling shock-enhanced mixing for scramjet combustor applications. Short combustor residence times, a requirement for fuel injection parallel to the freestream, and strong sensitivity of overall vehicle performance to combustion efficiency motivated the investigation. The following conclusions are supported by both experimental and computational results.

For the case of equal expansion and compression of the ramp surfaces, the ramp-generated vorticity and the shock-generated vorticity were of the same sign, order and scale. Shock-impingement led to seeding of streamwise vorticity directly on the injectant/air interface, whereas ramp-generated vorticity formed remote from the mixing interface. Baroclinic generation of vorticity at the base of the helium jet in the exit plane was the dominant factor in producing strong initial liftoff and complete separation of the jet from the surface.

The vorticity field coalesced into a counter-rotating vortex pair of a sense that caused the helium jet to migrate away from the surface. A constant jet trajectory slope of 0.025 was found for $\bar{x}>20$. Further, an increase in interfacial surface area and narrowing of species gradients was afforded by the strong convective forces. This led directly to increased mixing on a molecular scale through facilitation of diffusive processes. It is felt that the lack of spatial resolution in both the experiments and the computations led to a misrepresentation of the detailed form of the helium jet. Estimation of convective and diffusive phenomena showed that the internal structure of the jet may take the form of tightly wound, spiral species gradients. It is postulated that diffusion across these gradients in the near-field contributed significantly to molecular mixing. Farther downstream, molecular mixing was driven by turbulent processes which may have been enhanced by the largescale convection. Temporally-resolved planar Rayleigh imaging was used to provide qualitative insight into the scale and nature of the time-fluctuating flow component. The peak-to-peak spatial amplitude of the unsteady oscillations of image intensity about the mean was as large as four tenths of the injector height at $\bar{x}=10$. 
Experimentally, the peak helium mass fraction decay was proportional to $\bar{x}^{-1.37}$. For representative scramjet combustor conditions, it was projected that complete mixing of the hydrogen fuel to less than the stoichiometric mass fraction would require jet penetration into an area approximately 15 times the area of the injection plane. For the freestream velocities of this study, such behavior would be expected by $\bar{x} \approx 60$. Considerably more rapid mixing of helium to a similar mass fraction was displayed in the current effort due to significantly higher injectant to freestream static temperature ratios than would be expected in practice.

Several salient parametric dependencies have been investigated for the contoured wall fuel injectors. These will be discussed in a subsequent publication. ${ }^{7}$ The parameters investigated include injector spacing, combustor wall boundary layer height, and injectant to freestream pressure and velocity ratios.

\section{Acknowledgements}

Funding for this work was provided largely through NASA Grant NAG 1-842. The authors wish to thank many members of the technical staff at NASA Langley Research Center, particularly Dennis Bushnell, members of the Experimental Flow Physics Branch, and members of the Computational Methods Branch. The Rayleigh scattering data were obtained through a collaborative effort with B. Shirinzadeh, J. Balla, M. Hillard and R. Exton of the Instrument Research Division's Optical Spectroscopy Section. Supercomputing support was provided by the San Diego Supercomputer Center via a National Science Foundation Grant, the NASA Ames NAS facilities, the JPL/Caltech Cray, and the NASA Langley supercomputing facilities.

\section{References}

1. Kumar, A., Bushnell, D. M., and Hussaini, M. Y., "Mixing Augmentation Technique for Hypervelocity Scramjets," J. Propulsion, pp. 514-522, 1989.

2. Haas, F., and Sturtevant, B., "Interaction of Weak Shock Waves with Cylindrical and Spherical Gas Inhomogeneities," J. Fluid Mechanics, v. 181, pp. 41-76, 1987.
3. Yang, J., "An Analytical and Computational Investigation of Shock-Induced Vortical Flows with Applications to Supersonic Combustion," Ph.D. Thesis, California Institute of Technology, Pasadena, California, 1991.

4. Marble, F. E., Hendricks, G. J., and Zukoski, E. E., "Progress Toward Shock Enhancement of Supersonic Combustion Processes," AIAA Paper 87-1880, 1987.

5. Marble, F. E., Zukoski, E. E., Jacobs, J. W., Hendricks, G. J., and Waitz, I. A., "Shock Enhancement and Control of Hypersonic Mixing and Combustion," AIAA Paper 90-1981, 1990.

6. Waitz, I. A., "An Investigation of Contoured Wall Injectors for Hypervelocity Mixing Augmentation," Ph.D. Thesis, California Institute of Technology, Pasadena, California, 1991.

7. Waitz, I. A., Marble, F. E., and Zukoski, E. E., "A Systematic Experimental and Computational Investigation of a Class of Contoured Wall Fuel Injectors," submitted for presentation at the AIAA 30th Aerospace Sciences Meeting, Reno, Nevada, January 6$9,1992$.

8. Yeung, M-. T., unpublished results, California Institute of Technology, Pasadena, California, 1991.

9. Mcquaid, J., and Wright, W., "The Response of a Hot-Wire Anemometer in Flows of Gas Mixtures," Int. J. Heat and Mass Transfer, v. 16, no. 4., pp. 819-828, 1973.

10. Simpson, F. L., and Wyatt, W. G., "The Behavior of Hot-Film Anemometers in Gas Mixtures," J. Physics E: Scientific Instruments, v. 6., no. 10, pp. 968-1060, 1973.

11. Reis, V. H., and Fenn, J. B., "Separation of Gas Mixtures in Supersonic Jets," J. Chemical Physics, v. 39, no.12, 1963.

12. Chang, J. H., and Fenn, J. B., "Species Separation by Stagnation of Argon-Helium Mixtures in Supersonic Flow," Proceedings of the Seventh Rarefied Gas Dynamics Symposium, pp. 599-607, 1970.

13. Campargue, R., "The Separation Probe," Aerodynamic Separation of Gases and Isotopes, Lecture Series 82, von Karman Institute for Fluid Dynamics, 1976.

14. Shirinzadeh, B., Waitz, I. A., Balla, J., Hillard, M. E., Anders, J. B., and Exton, R. J., "Planar Rayleigh 
Scattering Results in Helium/Air Mixing Experiments in a Mach 6 Wind Tunnel," submitted to Optics Letters, 1991.

15. Shirinzadeh, B., Hillard, M. E., and Exton, R. J., "Condensation Effects on Rayleigh Scattering Measurements in a Supersonic Wind Tunnel," AIAA Journal, v. 29, no.2, pp. 242-246, 1991.

16. Shirinzadeh, B., Hillard, M. E., Blair, A. B., and Exton, R. J., "Study of Cluster Formation and Its Effects on Rayleigh and Raman Scattering Measurements in a Mach 6 Wind Tunnel," AIAA Paper 91-1496, 1991.

17. Carpenter, M. H., "Three-Dimensional Computations of Cross-Flow Injection and Combustion in a Supersonic Flow," AIAA Paper 89-1870, 1989.

18. Drummond, J. P., Carpenter, M. H., Riggins, D. W., and Adams, M. S., "Mixing Enhancement in a Supersonic Combustor," AIAA Paper 89-2794, 1989.

19. Eklund, D. R., Northam, G. B., and Fletcher, D. G., "A Validation Study of the SPARK Navier-Stokes Code for Nonreacting Scramjet Combustor Flowfields," AIAA Paper 90-2360, 1990.

20. Riggins, D. W., Mekkes, G. L., McClinton, C. R., and Drummond, J. P., "A Numerical Study of Mixing Enhancement in a Supersonic Combustor," AIAA Paper 90-0203, 1990.

21. Riggins, D. W., and McClinton, C. R., "A Computational Investigation of Flow Losses in a Supersonic Combustor," AIAA Paper 90-2093, 1990.

22. Kuo, K. K., Principles of Combustion, John Wiley and Sons, New York, pp. 357-359, 1986.

23. Cussler, E. L., Diffusion, Mass Transfer in Fluid Systems, Cambridge University Press, New York, pp. 108-111., 1984.

24. Broadwell, J. E., and Mungal, M. G., "Large-Scale Structures and Molecular Mixing," invited general lecture presented at the IUTAM Symposium on Fluid Mechanics of Stirring and Mixing, San Diego, California, 1990. 